\title{
PECEIVED
}

Aus 270986

OAK RIDGE

OSTI

NATIONAL LABORATORY

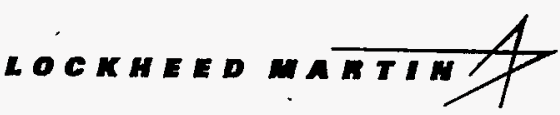

\section{A Study of Application Sensitivity to Variation in Message Passing Latency and Bandwidth}

Patrick H. Worley

Allen C. Robinson

David R. Mackay

Edward J. Barragy

MANAGED AMD OPERATED BY

LOCKHEED MARTN ENERGY RESEARCH CORPORATION FOR THE UNTED STATES DEPARTMENT OF ENERGY

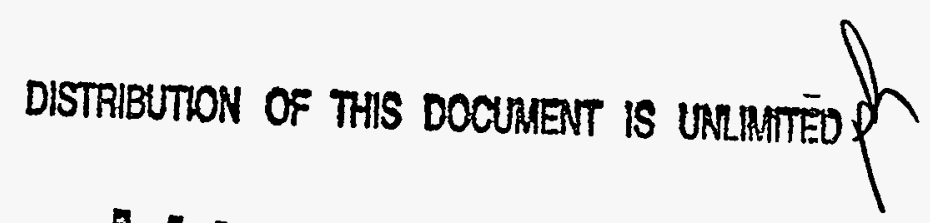


This report has been reproduced directly from the best available copy.

Available to DOE and DOE contractors from the Office of Scientific and Technical Information, P. O. Box 62, Oak Ridge, TN 37831; prices available from (423) 576-8401, FTS 626-8401.

Available to the public from the National Technical Information Service, U.S. Department of Commerce, 5285 Port Royal Road, Springfield, VA 22161.

This report was prepared as an account of work sponsored by an agency of the United States Government. Neither the United States Government nor any agency thereof, nor any of their employees, makes any warranty, express or implied, or assumes any legal liability or responsibility for the accuracy, completeness, or usefulness of any information, apparatus, product, or process disclosed, or represents that its use would not infringe privately owned rights. Reference herein to any specific commercial product, process, or service by trade name, trademark, manufacturer, or otherwise, does not necessarily constitute or imply its endorsement, recommendation, or favoring by the United States Government or any agency thereof. The views and opinions of authors expressed herein do not necessarily state or reflect those of the United States Government of any agency thereof. 


\section{DISCLAIMER}

Portions of this document may be illegible in electronic image products. Images are produced from the best available original document. 
Computer Science and Mathematics Division

Mathematical Sciences Section

\title{
A STUDY OF APPLICATION SENSITIVITY TO VARIATION IN MESSAGE PASSING LATENCY AND BANDWIDTH
}

\author{
Patrick H. Worley ${ }^{\dagger}$ \\ Allen C. Robinson • \\ David R. Mackay ${ }^{ \pm}$ \\ Edward J. Barragy **
}

+ Oak Ridge National Laboratory, Mathematical Sciences Section, P. O. Box 2008, Oak Ridge, TN 37831-6367

- Sandia National Laboratories, Computational Physics Research \& Development, 1431, P.O. Box 5800, Albuquerque, NM 87185-0819

* Oak Ridge National Laboratory, Center for Computational Sciences P. O. Box 2008, Oak Ridge, TN 378316203

** Intel Corp, CO1-02, 15201 NW Greenbrier Pkwy, Beaverton OR, 97006

Date Published: June, 1996

Research was supported by the Mathematical, Information and Computational Sciences Division of the Office of Computational and Technology Research Program, Office of Energy Research, U.S. Department of Energy

Prepared by the

Oak Ridge National Laboratory

Oak Ridge, Tennessee 37831 managed by

Lockheed Martin Energy Research Corp.

for the

U.S. DEPARTMENT OF ENERGY

under Contract No. DE-AC05-96OR22464 


\section{Contents}

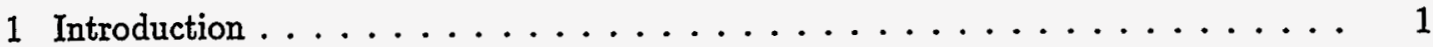

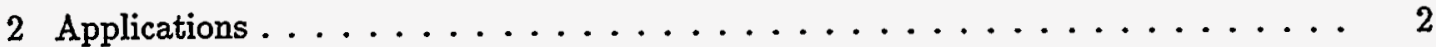

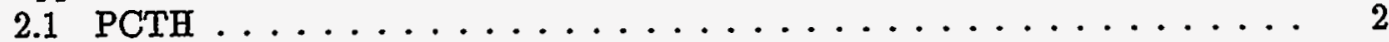

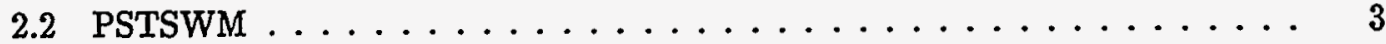

3 Methodology ............................. 4

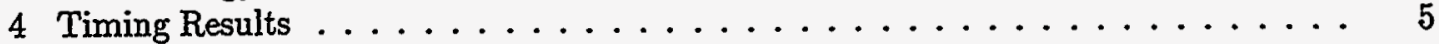

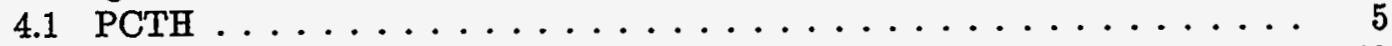

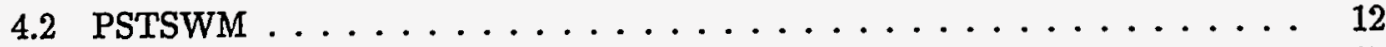

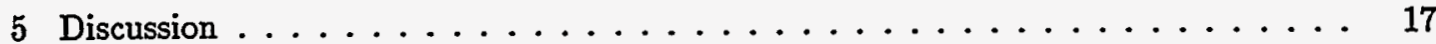

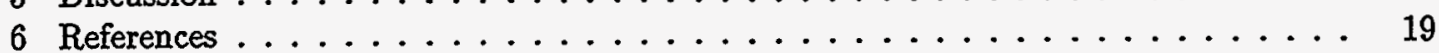




\title{
A STUDY OF APPLICATION SENSITIVITY TO VARIATION IN MESSAGE PASSING LATENCY AND BANDWIDTH
}

Patrick H. Worley

Allen C. Robinson

David R. Mackay

Edward J. Barragy

\begin{abstract}
This study measures the effects of changes in message latency and bandwidth for production-level codes on a current generation tightly coupled MPP, the Intel Paragon. Messages are sent multiple times to study the application sensitivity to variations in bandwidth and latency. This method preserves the effects of contention on the interconnection network.

Two applications are studied, PCTH, a shock physics code developed at Sandia National Laboratories, and PSTSWM, a spectral shallow water code developed at Oak Ridge National Laboratory and Argonne National Laboratory. These codes are significant in that PCTH is a "full physics" application code in production use, while PSTSWM serves as a parallel algorithm test bed and benchmark for production codes used in atmospheric moldeling. They are also significant in that the message-passing behavior differs significantly between the two codes, each representing an important class of scientific message-passing applications.
\end{abstract}




\section{Introduction}

Computing on massively parallel processing systems (MPPs) is sufficiently mature that large scale full physics applications codes are being run on machines with 1000 or more processors on a routine basis. These types of codes typically comprise over 100,000 lines of code and involve simulations of coupled physics across multiple length and time scales. MPPs provide access to the large amounts of memory and the compute power needed to complete these complex simulations at heretofore unprecedented levels of spatial resolution.

While "massively parallel" application codes have been designed to run on thousands of processors, there is still much to learn about their sensitivity to MPP design parameters such as latency, bandwidth, and network topology. In this study, we examine this sensitivity for two representative parallel applications, PCTH, a shock physics application developed at Sandia National Laboratories, and PSTSWM, a spectral shallow water code developed at Oak Ridge National Laboratory and Argonne National Laboratory. The information in this study is useful in optimizing machine design for cost performance and for scaling to tens of thousands of processors.

Performance studies of communication latency and bandwidth are most commonly used to characterize the peak or "peak achievable" performance on parallel platforms [3]. Such characterizations can be used to help explain the empirically observed performance difference across parallel platforms [6], [5] or between message passing libraries [15], or used to determine rates in performance models [1]. There has been significantly less work in characterizing the communication requirements of production-level parallel application codes, and, to our knowledge, almost no research on performance sensitivity to changes in latency, bandwidth, and topology. One reason for this lack is that performance modeling techniques that can accurately model network performance, like Markov models and Petri nets [9], [19], [20], are impractical to apply to full application codes running on hundreds or thousands of processors. The alternative is to do empirical studies using representative application codes.

The work closest in spirit to our research is the synthetic-perturbation technique developed at the National Institute of Standards [10], [11] and the performance studies by Rothberg [17], [18]. The synthetic-perturbation technique inserts idle loops into programs to determine the effect on overall performance of this type of perturbation, It was developed as a technique for identifying which routines are most important to tune when attempting to improve the performance of a parallel code. Rothberg used the similar technique of inserting idle loops in communication routines to evaluate sensitivity to network performance. As will be discussed later, inserting idle loops determines only the sensitivity to changes in message latency. Our study also investigates the effect of message passing bandwidth degradation and changes in topology. 
The outline of the paper is as follows. $\S 2$ describes the two applications. $\S 3$ describes the methodology used to measure sensitivity to the latency and bandwidth, as well as details on the computing environment in which the studies were made. $\$ 4$ describes the results of the experiments and initial interpretations. $\$ 5$ summarizes the results for the two codes and discusses the implications.

\section{Applications}

\subsection{PCTH}

PCTH was developed at Sandia National Laboratories and is a parallel three-dimensional shock wave physics code currently being used as a production simulation tool [16]. The code is a parallel version of the CTH shock physics code, a highly regarded modeling tool in the DoD and DOE modeling community, that models large-deformation material motion arising from high explosive detonation or high velocity impacts [14].

The algorithm implemented in the code solves equations modeling the conservation of mass, momentum and energy through a two stage process. The first stage models the change in physical quantities as seen in the frame of moving material. The second phase is a remap of these quantities back to the original fixed grid. Interface reconstruction algorithms are implemented to transport mixed material cell quantities in such a way as to maintain material interfaces. The material constitutive models implemented in the code include a von Mises elastic, perfectly-plastic yield stress models, a pressure-based fracture model, and a fracture model based on principal stress. Both analytic and tabular material equation of states as well as techniques for modeling of high-explosive materials are available in the code. These capabilities allow the simulation of a large range of important problems.

PCTH was developed specifically for message-passing parallel computers and is easily ported to any message-passing architecture. For example, the MPI and PVM message-passing interface libraries are standard build options for the PCTH code. The parallel version of the code is relatively simple in terms of its message-passing complexity. A three dimensional domain decomposition is used to break the three dimensional domain of interest into subdomains, each of which contains typically $5-20$ thousand computational cells. One subdomain is assigned to each processor in the MPP ensemble. Each surface of a subdomain is augmented with a one cell deep layer of "ghost" cells. Ghost cells are used to store information communicated from adjacent processors. For the regular Cartesian mesh problems considered in this study, each interior cubical subdomain has 6 neighbors corresponding to the 6 plane faces. Communication with the additional 20 corner and edge neighbors is accomplished by including the ghost cell information on the four edges of a communication plane in each boundary update and updating 
in one direction at a time. Tracer particles track points attached to the flow and are thus required to "move" from processor to processor. Global operations include a global minimum for time step limiting and global sums for conservation checks.

\subsection{PSTSWM}

PSTSWM is a parallel algorithm testbed and benchmark code developed at Oak Ridge National Laboratory and Argonne National Laboratory that solves the nonlinear shallow water equations on a rotating sphere using the spectral transform method [13]. The nonlinear shallow water equations are a two-dimensional atmospheric-like fluid prediction model that exhibits many of the features of more complete models and is commonly used to investigate numerical methods [2]. The spectral transform algorithm of the code follows closely how CCM2, the Community Climate Model developed at the National Center for Atmospheric Research (NCAR), handles the dynamical part of the primitive equations [7], and the parallel algorithms implemented in PSTSWM include those currently used in PCCM2, the message-passing parallel implementation of CCM2 [4]. PSTSWM is used to evaluate the performance of parallel platforms for spectral atmospheric circulation models and to develop new parallel algorithms for use in PCCM2 [5], [6].

PSTSWM is a parallel implementation of the sequential code STSWM [8] developed at NCAR that has subsequently been modified to add vertical levels to the code, allowing the parallel algorithms to better capture the requirements and performance for three dimensional models like PCCM2. PSTSWM also has embedded 6 of the 7 test cases specified by Williamson et al. [22] to test the ability of numerical methods to simulate important flow phenomena.

The spectral transform is the most complicated portion of a spectral atmospheric circulation code to parallelize, and the performance is sensitive to how it is done. During each timestep of the model simulation, the state variables of the problem are transformed between the physical domain, where most of the physical forces are calculated, and the spectral domain, where the terms of the differential equations are evaluated. The physical domain is a tensor product longitude-latitude-vertical level grid. The spectral domain for a given vertical level is the set of spectral coefficients in a spherical harmonic expansion of the state variables. Transforming from physical coordinates to spectral coordinates involves performing a fast real Fourier transform for each line of constant latitude, followed by integration over latitude using Gaussian

quadrature (approximating the Legendre transform) to obtain the spectral coefficients. The inverse transformation involves evaluating sums of spectral harmonics and inverse real FFTs, analogous to the forward transform.

PSTSWM includes many different algorithms and data distributions for the spectral transform. Two basic alternatives are available for both the Fourier and Legendre transforms: 
distributed algorithms, using a fixed data decomposition and computing results where they are assigned, and transpose algorithms, remapping the domains to allow the transforms to be calculated sequentially. Multiple implementations are supported for each type of algorithm and the assignment of processors to transforms is also determined by input parameters. For example, input parameters specify a logical 2-D processor grid and define the data decomposition of the physical and spectral domains onto this grid. If 16 processors are used, these can be arranged as a $4 \times 4$ grid, an $8 \times 2$ grid, a $16 \times 1$ grid, a $2 \times 8$ grid, or a $1 \times 16$ grid. This specification determines how many processors are used to calculate each parallel Fourier transform and how many are used to calculate each parallel Legendre transform. There are also options for specifying how data is exchanged between nodes, including whether to try to overlap communication and computation, and what message-passing interface to use [23].

For the results presented in this paper, two different parallel algorithms were examined: a transpose Fourier transform/transpose Legendre transform algorithm and a transpose Fourier transform/distributed Legendre transform algorithm. Previous studies have shown these algorithms to be among the best performers across a range of platforms and problem sizes. For this study, these algorithms are also interesting because of their different communication patterns and costs. The transpose Legendre algorithm involves an all-to-all personal exchange across each logical processor column, with each processor sending distinct medium-sized messages to every other processor in its column. The distributed Legendre algorithm uses a ring algorithm to implement a distributed vector sum, sending the same number of messages as the transpose algorithm but sending only to nearest neighbors and sending larger messages when using moderate to large numbers of processors. Table 1 summarizes the number of messages and the total message volume per timestep for each node when using a $P_{X} \times P_{Y}$ logical processor grid. Here $N$ is the number of grid points in the three dimensional physical domain grid. The message count and volume are functions of the problem size and of the number of processors, and the specifics will be given in the results sections.

\begin{tabular}{|l|r|r|}
\hline Algorithm & message count & message volume (words) \\
\hline Transpose FFT & $2\left(P_{X}-1\right)$ & $13 N /\left(P_{X} P_{Y}\right)$ \\
Transpose LT & $2\left(P_{Y}-1\right)$ & $(26 / 3) N /\left(P_{X} P_{Y}\right)$ \\
Distributed LT & $2\left(P_{Y}-1\right)$ & $(4 / 3) N / P_{X}$ \\
\hline
\end{tabular}

Table 1: Number and total volume of messages for the different transform algorithms.

\section{Methodology}

In the present study, net bandwidth is defined as the time to complete, or receive, the message, including latency, bandwidth, software, and hardware effects. The approach taken is to degrade available net bandwidth and observe the impact on application performance. 
Two approaches are used to degrade net bandwidth. The first is to send and receive each message multiple times. For message types where subdomain information is exchanged, this procedure has the important effect of "consuming" hardware bandwidth. That is, contention effects in the 2D mesh interconnect are preserved as bandwidth is degraded. Various levels of degradation are achieved by increasing the repeat count on the number of times each message is sent/received. Global sums and minimums are repeated a fixed number of times also. This methodology has the limitation that the message repetition process may consume main processor execution time. This is only a factor for codes that effectively overlap computation and communication. The effect is felt to be minimal and is a penalty that could not be avoided. As a check, this technique was applied to the message passing swap routine from PSTSWM in a stand alone test using two processors. The performance results were consistent as the message count increased, showing an approximately linear increase in execution time, giving confidence in using this method for decreasing available net bandwidth.

The second approach used to degrade net bandwidth is to degrade message latency by inserting spin waits in the communication routines. Various levels of degradation are achieved by varying the time spent in the spin wait, similar to the approaches used in [11], [17], and [18].

All simulations were run on Intel Paragon systems under the SUNMOS operating system [12], [21]. SUNMOS is used in these experiments rather than the vendor-supplied OSF node operating system because SUNMOS provides lower latency, higher bandwidth, and uses less memory, allowing more of the parameter space to be examined. The Intel Paragon uses a 2D mesh interconnect topology connecting "compute nodes" containing two or three processors. The basic message-passing performance parameters are shown in Figures 1 and 2. Unidirectional message-passing rates are shown first and peak at approximately $160 \mathrm{MB} / \mathrm{s}$. Bidirectional rates are approximately twice the unidirectional rates. Figure 2 shows transfer times for relevant values of message length, indicating a zero-length message latency of 17 microseconds. Beyond $8 \mathrm{~KB}$ message sizes, the slope of the transfer time curve is roughly constant.

\section{Timing Results}

\subsection{PCTH}

PCTH timing results are presented for a basic test problem simulating the impact of a copper ball on an inclined steel plate. Results are presented for the first 3.5 microseconds of simulation time. PCTH performance numbers are given as a "grind" time. This is the computational time divided by the total number of cells and the total number of cycles. Thus the grind time represents a measure of the time for the code to advance a single computational cell one time step. The current average for any simulation time is from the start of the simulation and is 


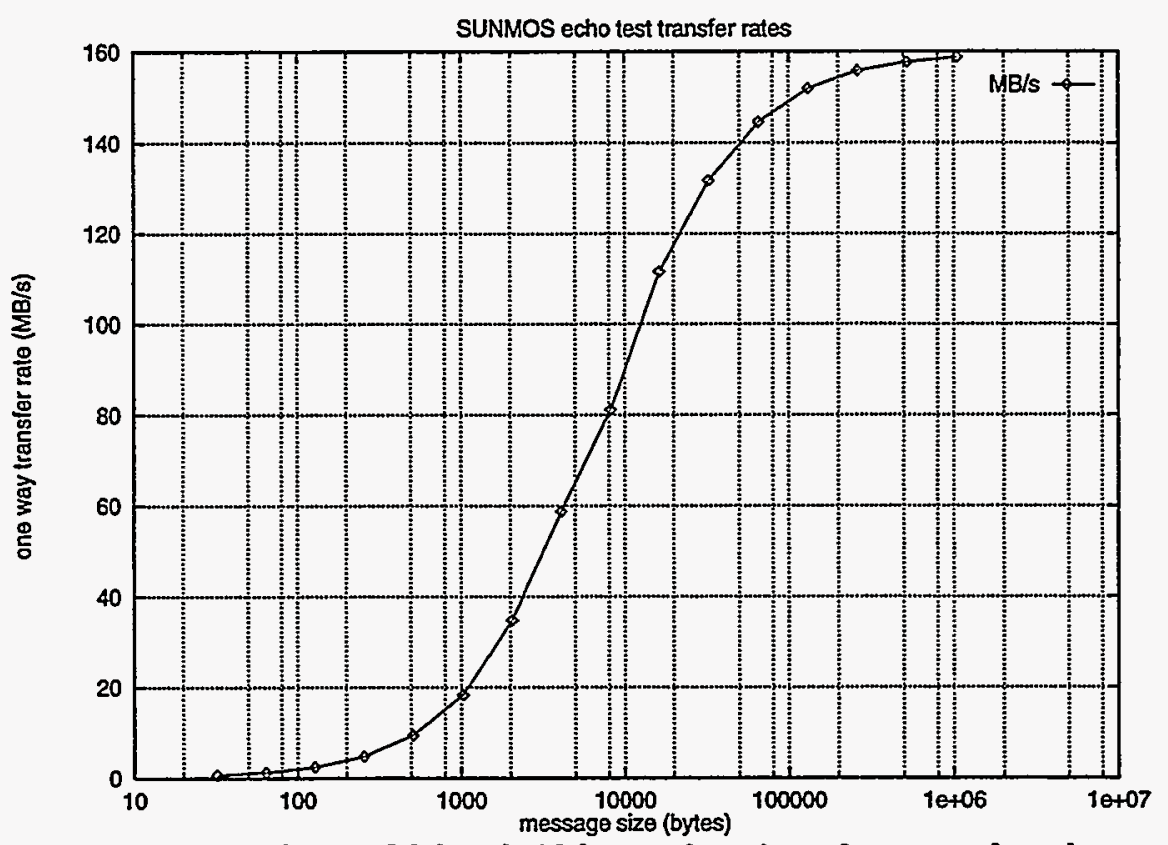

Figure 1: Net SUNMOS bandwidth as a function of message length.

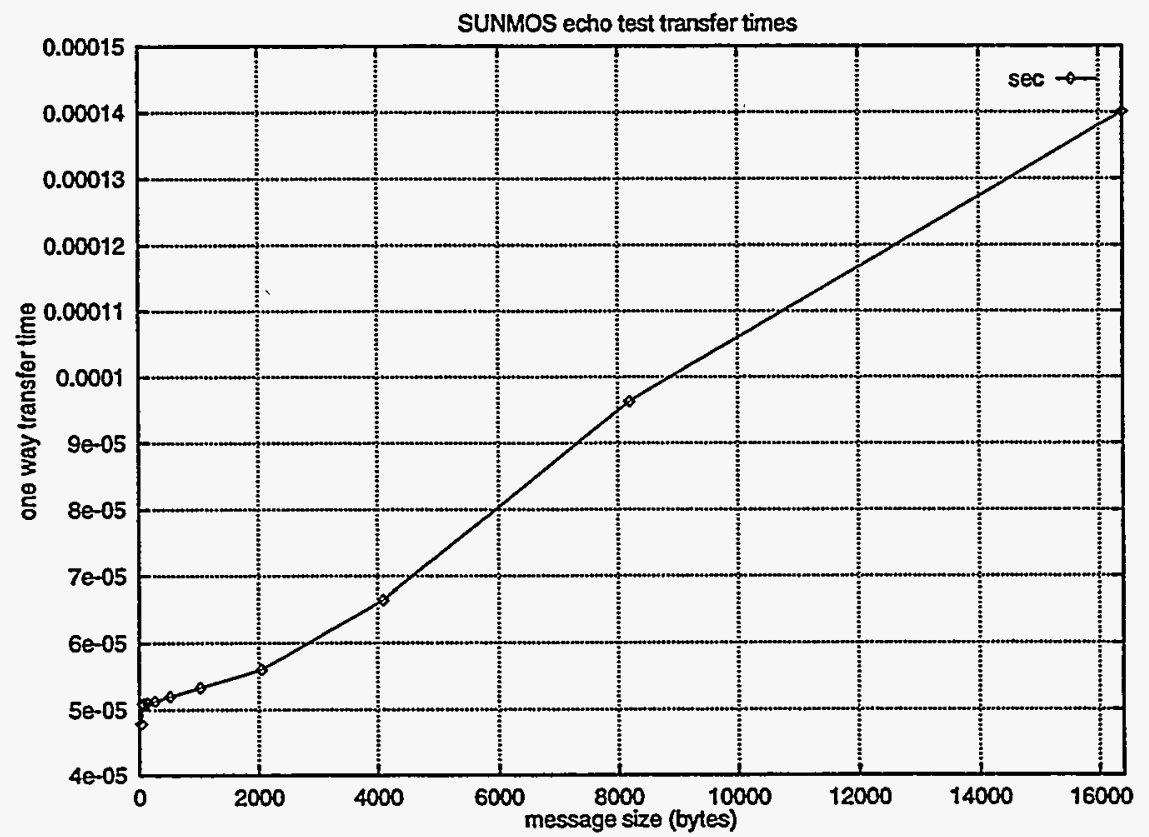

Figure 2: Net SUNMOS transfer time as a function of message length. 
taken over all cells. There is an increase in the grind time values starting at 0.4 microseconds of simulation time. This is when the initial contact between the two objects occurs, and large deformation increases the CPU time per time step.

Six different analyses of PCTH performance are presented, followed by a discussion of the results. The first analysis indicates the effect of varying the message repeat count, $R$, for a given large problem running on a fixed number of processors. Second, a fixed-size speedup analysis is presented for a moderate sized problem for different values of the repeat count. Third, the effect of $R$ on scaleup performance is investigated, wherein the number of computational cells per processor is fixed as the number of processors is increased. Fourth, estimates of network contention are presented. Fifth, the effects of bandwidth degradation are investigated for differing fractions of memory use per compute node for a fixed problem size. This is an attempt to examine trade offs of the amount of memory per compute node versus the total number of compute nodes as bandwidth is degraded. Sixth, the effect on performance of degrading latency alone is investigated.

Fixed problem analysis. Figure 3 shows the variation in grind times for an 8 million cell problem running on 512 nodes of the Paragon. This results in roughly 16,000 cells per node, which is sufficient to fill memory on a $16 \mathrm{MB}$ compute node. On average, each processor sends/receives 333 messages per time step, with an average message length of approximately $6 \mathrm{~KB}$. Grind times for message repeat counts of 1 to 128 are shown.

For repeat counts of 1,16 and 128 , the grind times at 1.45 microseconds of simulation time in Figure 3 are 3.68, 4.03 and 7.29 . This implies an overhead of $9.5 \%$ and $98.1 \%$ respectively. Thus a 16 fold reduction in net bandwidth results in only a $10 \%$ increase in overall execution time. For the scaling of computation and communication found in PCTH, a larger problem, run on compute nodes with more than $16 \mathrm{MB}$ of memory, would see even less impact from the bandwidth degradation.

Fixed problem size analysis. Fixed problem size results are shown in Figure 4 for a 1 million cell version of the copper ball steel plate problem. Grind time data at time step 50 is compared as the number of processors is increased. The resulting curves are shown for message repeat counts $R$ of 1,8 , and 64. An ideal speedup curve is shown for comparison which drops from $20 \mu \mathrm{sec}$. to $2.5 \mu \mathrm{sec}$. as the number of processors increases from 64 to 512 . This problem requires a minimum of 64 processors to run on 16MB compute nodes. Therefore, the ideal curve and the following efficiency results are calculated relative to the grind times for a 64 processor run. For $R=1$, relative efficiency is $63 \%$ on 512 nodes. For a repeat count of $R=64$, this relative efficiency drops to $44 \%$. Note that this problem is quite small for 512 processors, requiring only $12.5 \%$ of the 16 Mbytes of available memory, and a heightened sensitivity to message-passing 


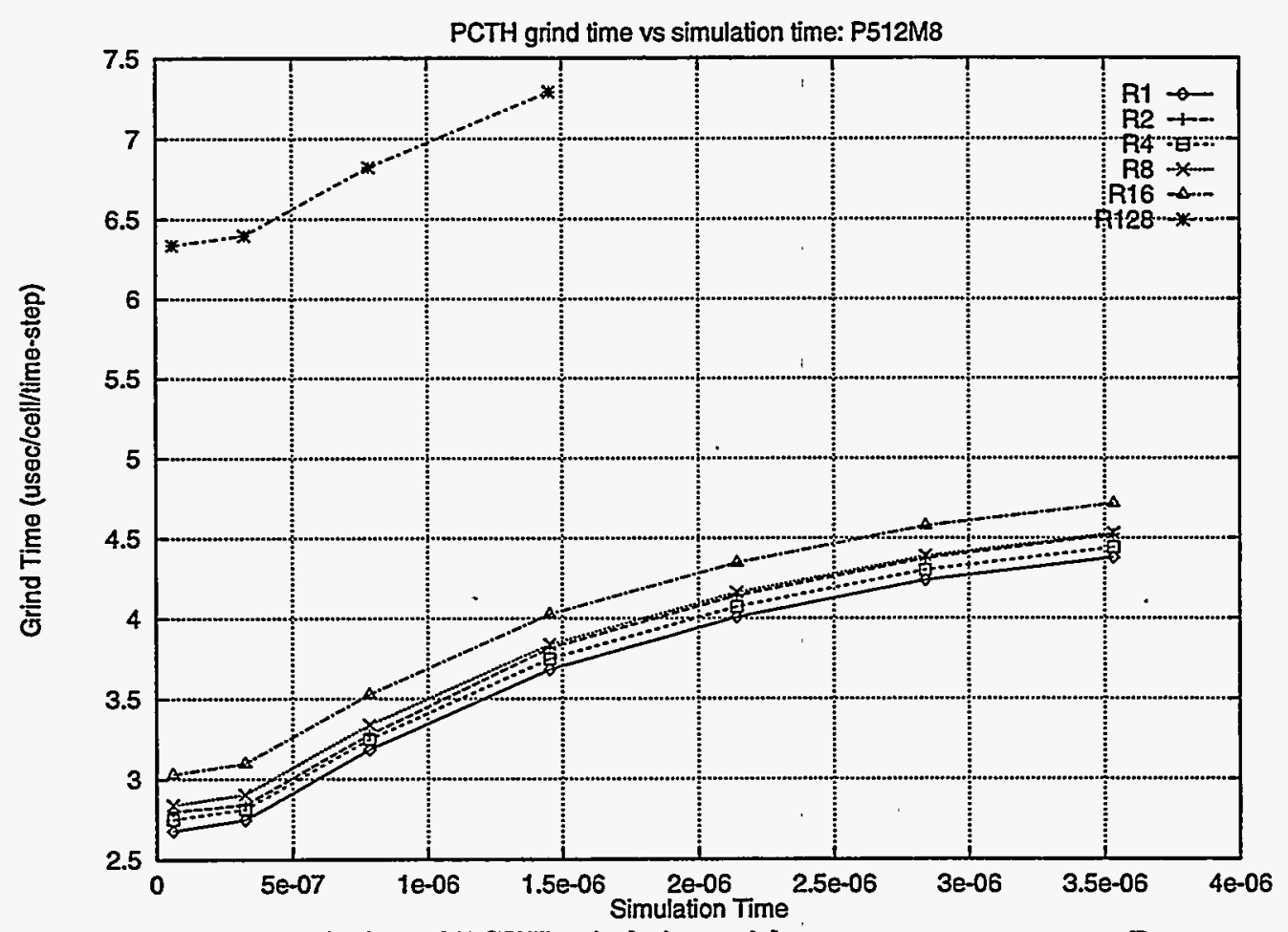

Figure 3: Variation of PCTH grind time with message repeat count, $R$.

performance would be expected.

Scaled problem size analysis. In production use, PCTH is often run in a scaleup mode of operation. An initial simulation might be run at a moderate resolution, perhaps 1 million cells. This would be followed by a high resolution simulation using 8 million cells. In both cases, memory on a compute node is always filled and a number of processors is selected to match the total problem size. Figure 5 shows the wall clock time per time step (at a simulation time of roughly 0.06 microseconds) for three problem sizes: 128,000 cells, 1 million cells and 8 million cells. The increase in the cell count by a factor of 8 between each problem corresponds to a uniform refinement in each spatial coordinate. Each problem is run with 16,000 computational cells per processor at ensemble counts of 8,64 and 512 processors respectively. Run time values for repeat counts of 1,16 , and 128 are shown. Comparing run time values for 1 million and 8 million cells shows modest increases for any given repeat count. Most of this increase can be attributed to the relative increase in the number of interior subdomain faces versus exterior faces, which require no communication. Thus only for 512 processors at the 8 million cell problem is the asymptotic distribution of interior versus exterior faces reached. The effect is particularly pronounced going from 8 to 64 processors in the 128 thousand and 1 million cell timings. 


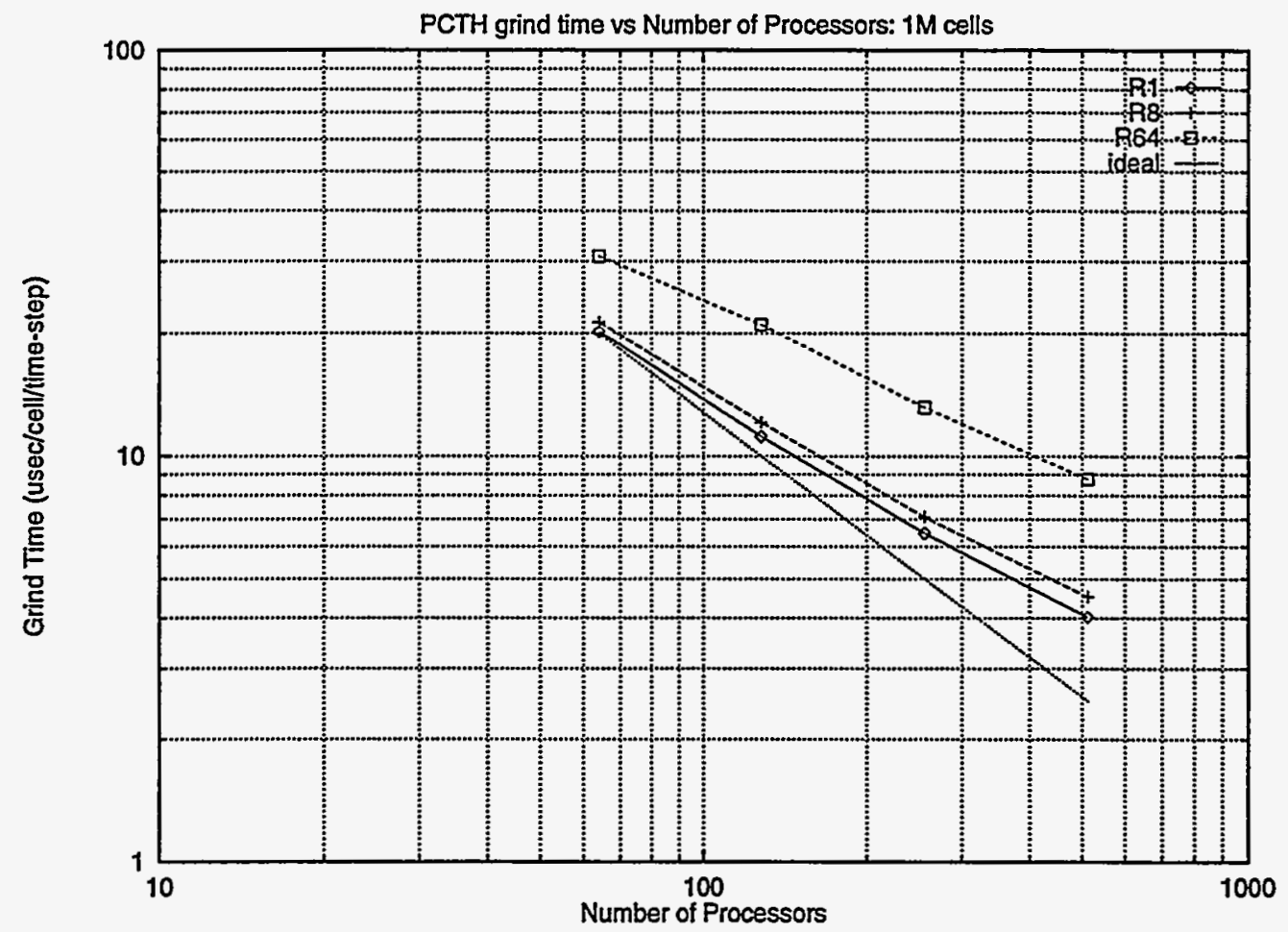

Figure 4: Variation of PCTH grind time with number of processors at time step 50 for various message repeat counts, $R$.

Contention estimate. For PCTH running under SUNMOS, contention can be measured indirectly by timing individual message sends in the application. Maximum and minimum values for sends are not affected by the types of load imbalances present in PCTH or by other performance issues present within the message receive software. Table 2 shows values for the average (over the processors) maximum and minimum send times for the 1 million cell problem running on 64 processors and the 8 million cell problem running on 512 processors. The ratio of the maximum and minimum is a measure of the contention. The Table shows only the minimum values observed over a sequence of time steps. The observed average maximum values varied quite drastically ( 2 fold) when the machine load changed. As indicated, the data shows a ratio of about 6.5 for send times.

\begin{tabular}{|l|c|c|c|}
\hline & Avg Max Send & Avg Min Send & Ratio \\
\hline I million cells & 505 & 82 & 6.7 \\
8 million cells & 495 & 76 & 6.5 \\
\hline
\end{tabular}

Table 2: Average $\mathrm{Max} / \mathrm{Min}$ message send times as problem size is scaled up $\mathrm{R}=1$.

Fixed memory size per node analysis. Of fundamental interest in MPP machine design is the question of how application performance varies as the memory per compute node and the interconnect bandwidth are varied. This question is examined by fixing the problem size at 


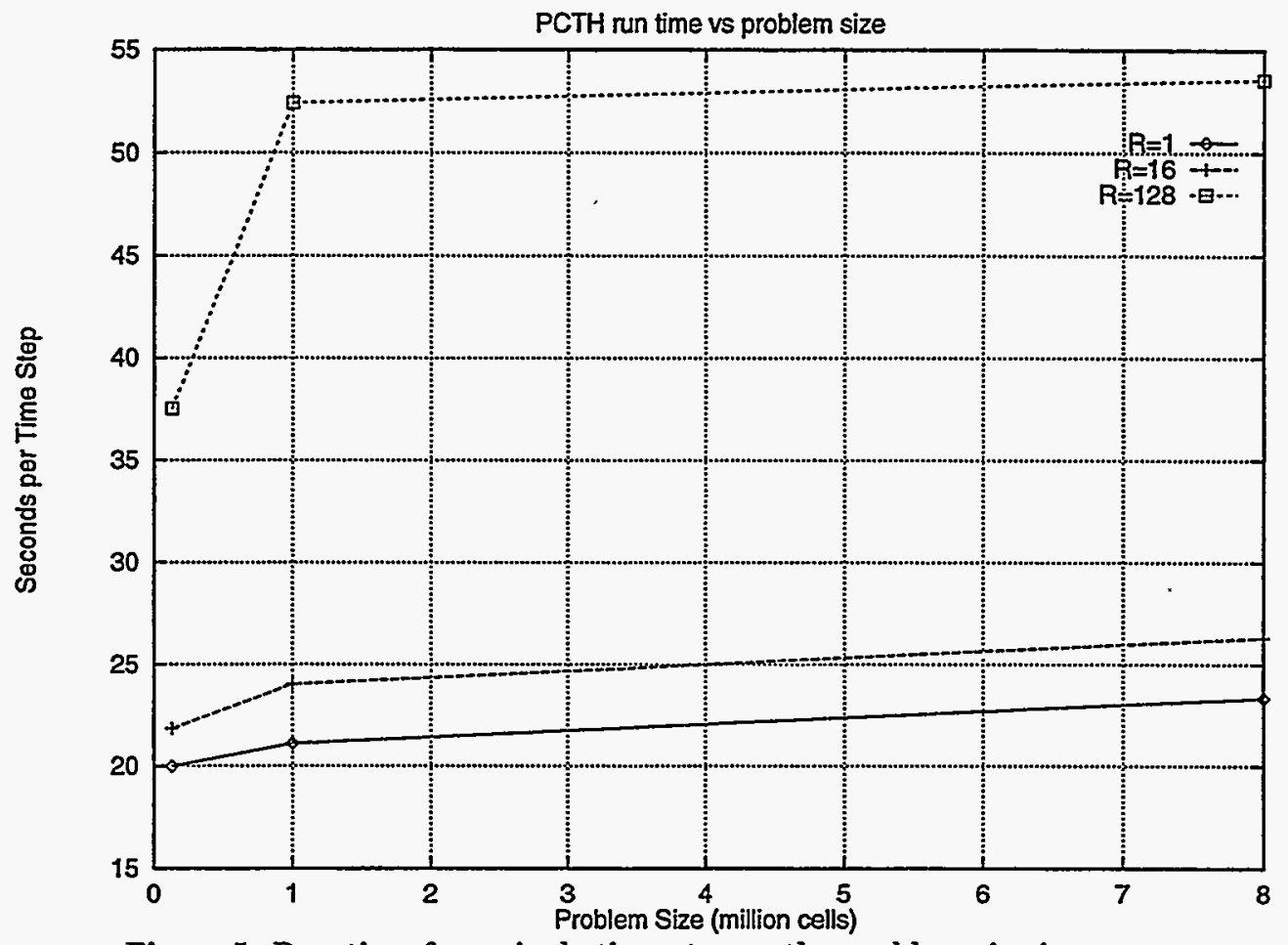

Figure 5: Run time for a single time step as the problem size increases.

1 million cells and running on 64, 128 and 256 processors for a range of message repeat counts. This corresponds to varying the fraction of available memory used on each processor. For 64 processors, one obtains 16,000 cells per processor and represents $100 \%$ memory use on a $16 \mathrm{MB}$ compute node. Similarly, 128 and 256 processors correspond to $50 \%$ and $25 \%$ memory use. Figure 6 shows relative grind times at time step 25 for various repeat counts for the 1 million cell problem. One curve is shown for each level of memory use: $25 \%, 50 \%$ and $100 \%$. The data shows essentially linear growth in the relative timings as the repeat count is increased. The slope of the curves increase as the memory use decreases, as expected. To quantify the effects of design tradeoffs on performance, consider the case of $R=32$. Here one sees a roughly $25 \%$ performance loss when memory is filled (16MB compute node). Effectively dropping the compute node size to $4 \mathrm{MB}$ results in a $50 \%$ performance loss. Thus, the following tradeoff occurs: reducing the amount of memory per node may allow for more nodes given a fixed machine cost, which in turn may allow faster overall application execution, but at the cost of greater demands on network performance. Of course as memory is reduced one must also look at the loss of total available memory to object code storage and determine at what point required maximum available user memory will take priority over turn around time.

Bandwidth degradation techniques. In the results presented so far a question may arise as to the strategy used for degrading bandwidth and latency. Thus far, this has consisted 


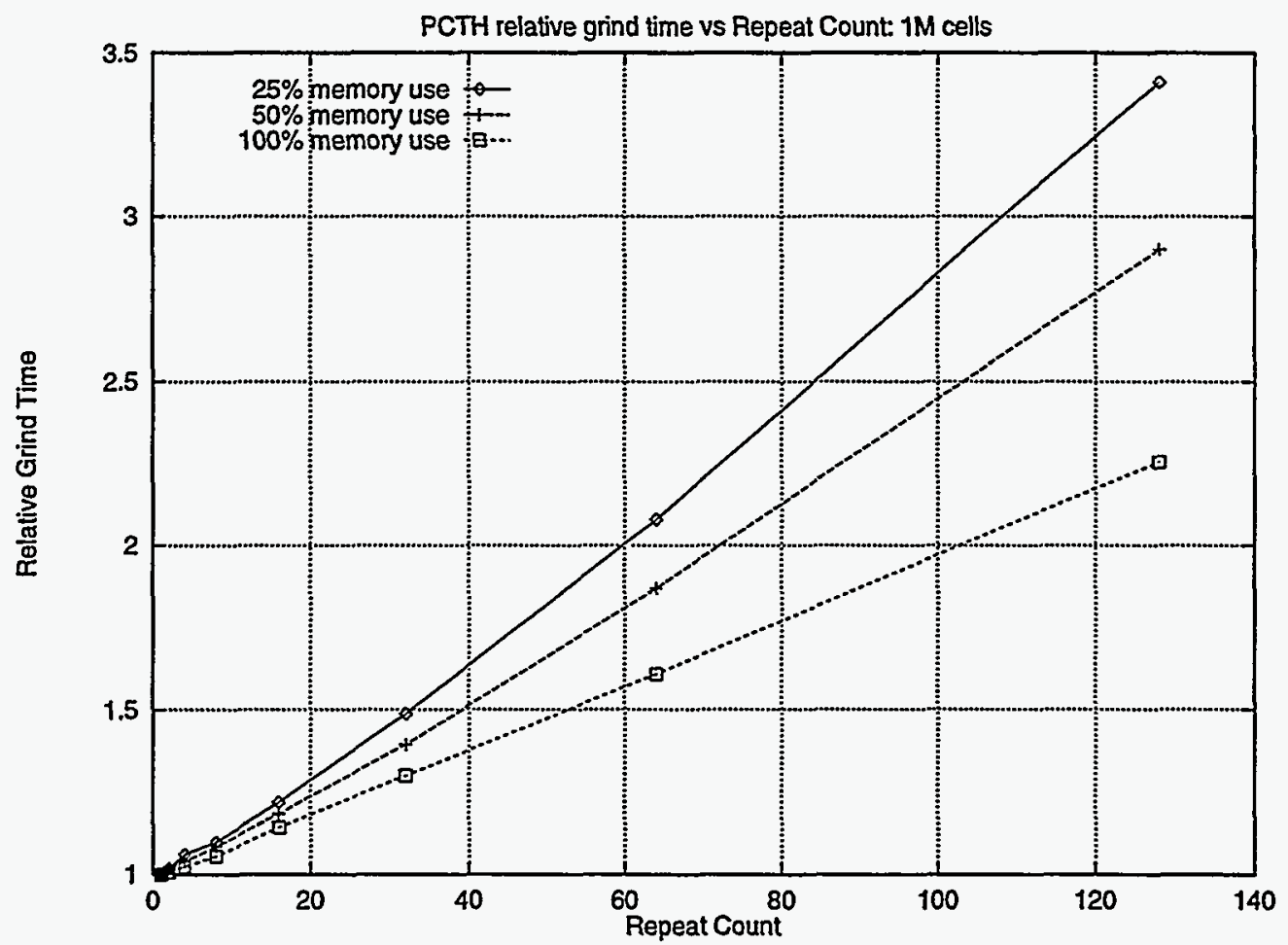

Figure 6: Variation of PCTH run time with message repeat count, $R$ for various levels of memory use.

of simply sending/receiving each application message multiple times. The argument used to justify this strategy is that available hardware bandwidth is consumed. An alternative approach is to insert delays before each message send and each message receive. This may be regarded as degrading latency alone. It has been observed that for the 1 million cell problem, the minimum time to send a message is about 70 microseconds, while the minimum receive time is about 120 microseconds. This gives an average of about 100 microseconds for each. Thus, contention effects aside, a repeat count of 16 is roughly equivalent to inserting a latency delay of $16 \cdot 100=1600$ microseconds in both the send and receive. Figure 7 shows grind time values at time steps 25 and 50 for message repeat counts of $R=1,16,128$. It also shows grind times when latency delays of 1600 and 12800 microseconds are inserted for both sends and receives at a repeat count of $R=1$. As is clearly shown, the performance degradation due to increased latency is about one third of that obtained by repeating messages. This indicates that accounting for contention effects is important when exploring application sensitivities. The multiple message technique is clearly superior to the message delay technique in this regard.

Discussion. Results for PCTH performance have demonstrated a number of points. First it is clear that PCTH could easily tolerate bandwidth reductions of an order of magnitude without impacting performance more than $10 \%$, given the scaleup mode of operation used in production. This is important in terms of machine design given that interconnect cost is directly related to 


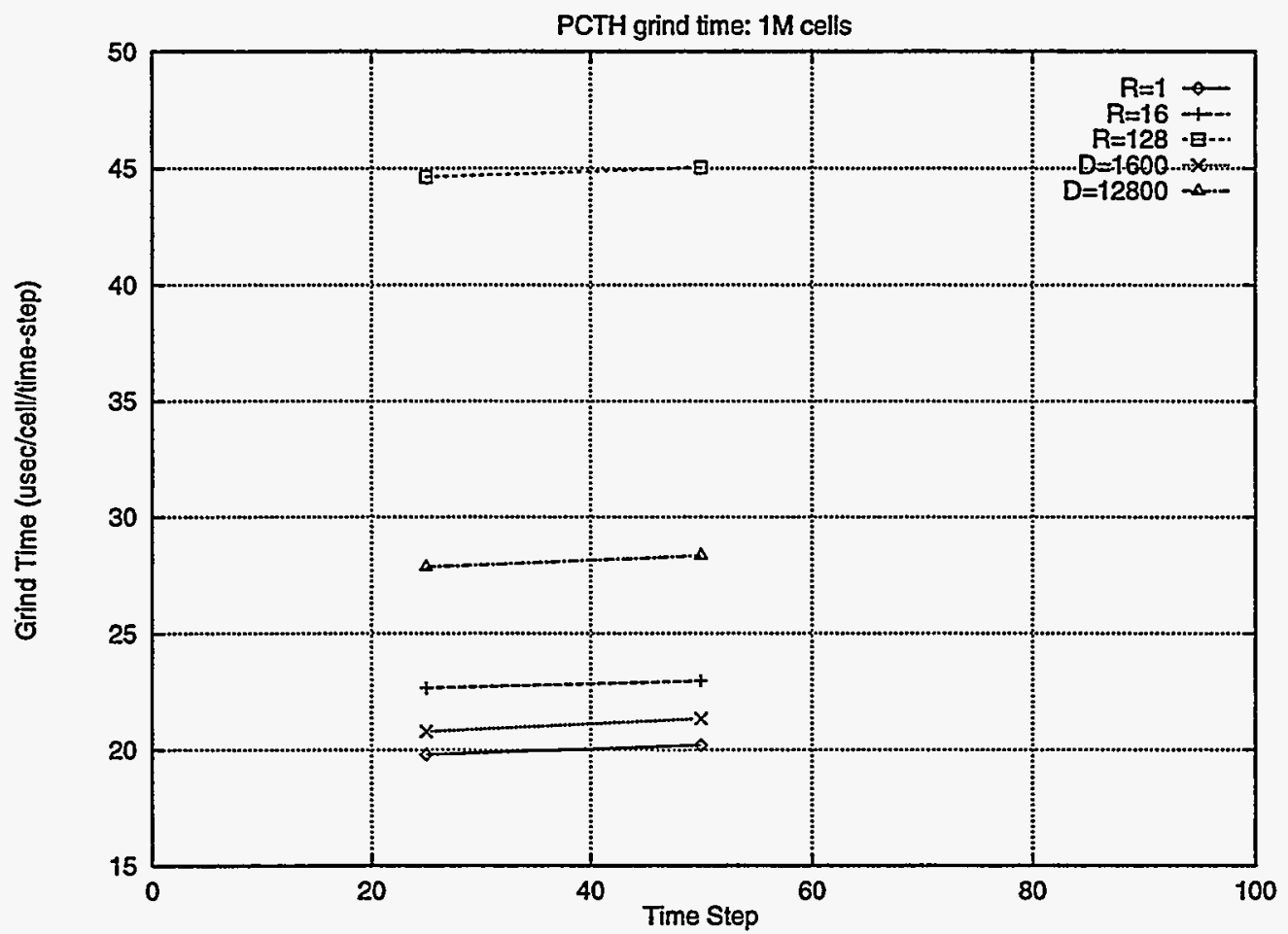

Figure 7: Variation of PCTH grind time with message repeat count, $R$ and latency delay, D.

the bandwidth. From another point of view, it seems clear that the current Paragon interconnect coupled with a more powerful compute node would be more than adequate for applications like PCTH. Next, it would appear that message contention on the $2 \mathrm{D}$ interconnect generated by 3D application communication patterns is a significant scaling factor for overall communication performance. The cost/performance tradeoffs of a $2 \mathrm{D}$ versus $3 \mathrm{D}$ interconnect are not clear. The results also seem to indicate that for the current system, reasonable performance could be obtained for PCTH running on current compute nodes with less than $16 \mathrm{MB}$ of memory.

\subsection{PSTSWM}

Changing the problem size in meteorological models is not undertaken lightly. At the minimum, the physical parameterizations must be reexamined, and there remains the question of acquiring appropriate input data for a given model resolution. While PSTSWM is not limited to a fixed size problem, issues of performance tuning for parallel meteorological models should be viewed from a fixed problem size point of view due to typical production requirements.

PSTSWM timing results are presented for two representative problem resolutions: T42L16,

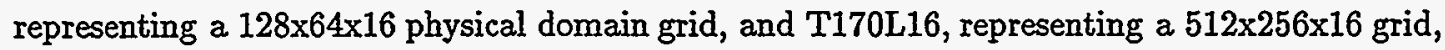
and for 22 time steps, representing 12 hours and 3 hours of simulated time for T42L16 and T170L16 respectively. There are no simulation time- or solution-dependent load imbalances in PSTSWM, so this relatively short simulation is sufficient to measure the performance. For 
these experiments, we use embedded problem option \#4: a forced low in the jet stream. The forcing in this option simulates the computational cost of the physical forcing found in full global atmospheric circulation models.

We present results for the two parallel algorithms described previously, a transpose FFT combined with a transpose Legendre transform and a transpose FFT combined with a distributed Legendre transform. In the initial results, the domain decomposition is over a logical square processor grid of size $4 \times 4,8 \times 8$, or $16 \times 16$. The physical layout of the nodes was also a square grid that matched the logical grid.

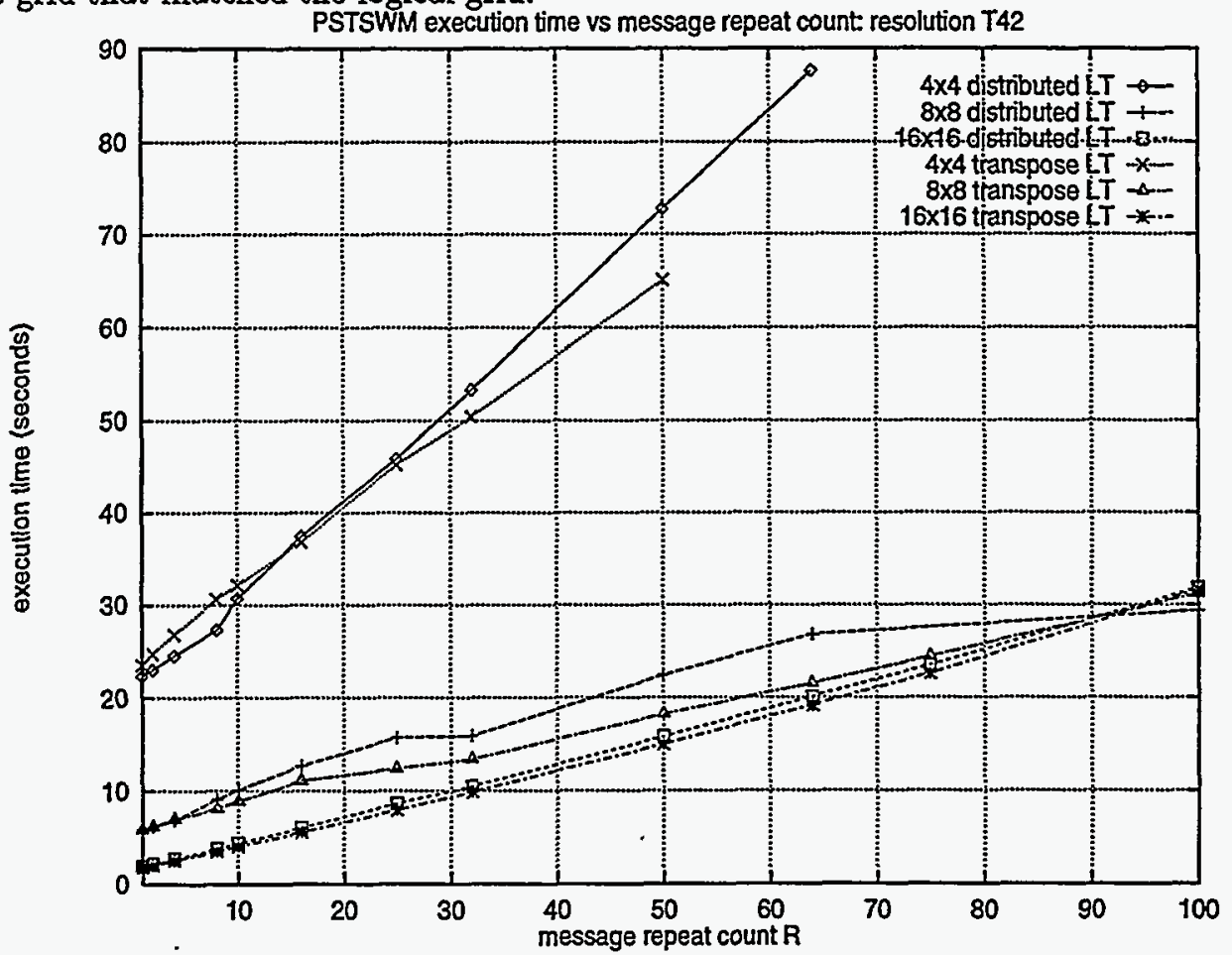

Figure 8: Variation of execution time for PSTSWM at resolution T42L16 for different number of processors as message repeat count $R$ increases.

Figure 8 shows execution times for resolution T42L16 on 16, 64, and 256 compute nodes plotted against the repeat count, $R$, for the messages. Figure 9 shows similar results for resolution T170L16. Notice the steeper slope in Figures 8-9 for the cases running on $4 \times 4$ compute node grids. As can be seen from Table 1, message volume per node is a decreasing function of the number of nodes, and the performance on smaller node configurations is more sensitive to bandwidth reduction. Timings on the $16 \times 16$ grid for T170L16 using the transpose IT algorithm for message repeat counts of 1 and 10 are 36.7 and 55.0 seconds, respectively. This corresponds to a 50 percent performance decrease for a factor of 10 decrease in message bandwidth.

In Figure 10, we compare the effect of net bandwidth on efficiency. This graph utilizes 


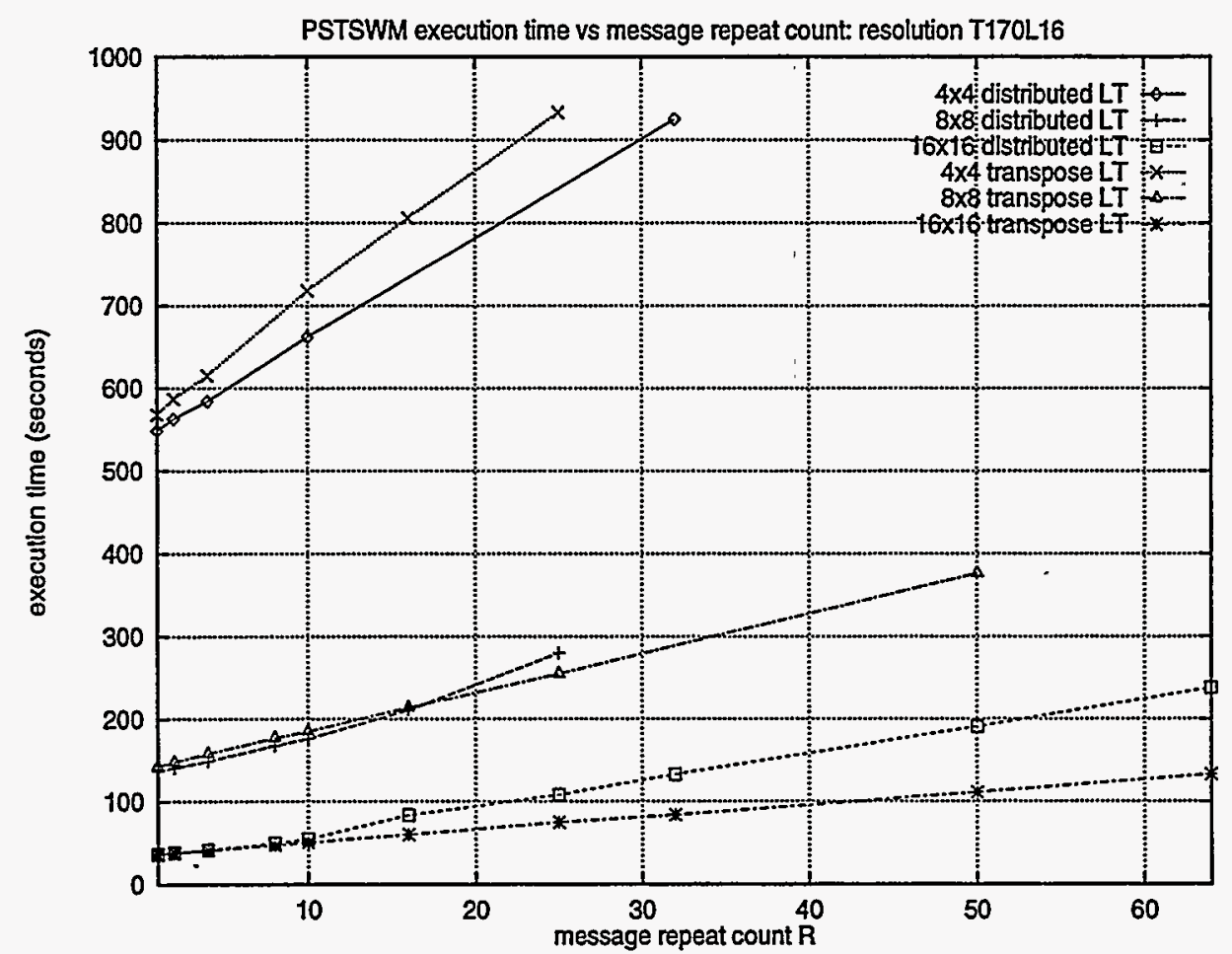

Figure 9: Variation of execution time for PSTSWM at resolution T170L16 for different number of processors as message repeat count $R$ increases.

data from T170L16 runs using the transpose LT parallel algorithm. The compute time on a $4 \times 4$ compute mesh without any message repeats is the base reference value (efficiency $=1.0$ ). We compute efficiency for the various grid layouts with different numbers of messages repeats. Notice that without repeating messages the efficiencies are all above 0.95 . As the $R$ count increases, efficiency decreases. For example, at an $R$ count of 16 , efficiency has dropped below 0.60 on 256 processors. As available net bandwidth decreases, PSTSWM does not scale well on additional processors for this problem size.

We also ran tests adding constant delays in an idle loop in the message-passing routines. These tests showed that any increase in latency had to be on the order of magnitude of several milliseconds to significantly affect performance, which is much higher than current parallel computers require. More important was what the latency or delay tests showed about contention. At resolution T170L16 on a $4 \times 4$ grid of compute nodes the largest message sent is 2 MBytes. Based on message echo tests, it takes 12739 microseconds to send a message of this length on the Paragon. Within the FFT and the LT all messages are the same length, but the message lengths in the FFT are not the same as the message lengths in the LT. By using the maximum message length (from FFT), we slightly underestimate the magnitude of the difference between the message repeat and message delay approaches in the following discussion.

To test the influence of mesh contention we equate every 12.739 millisecond message delay to 


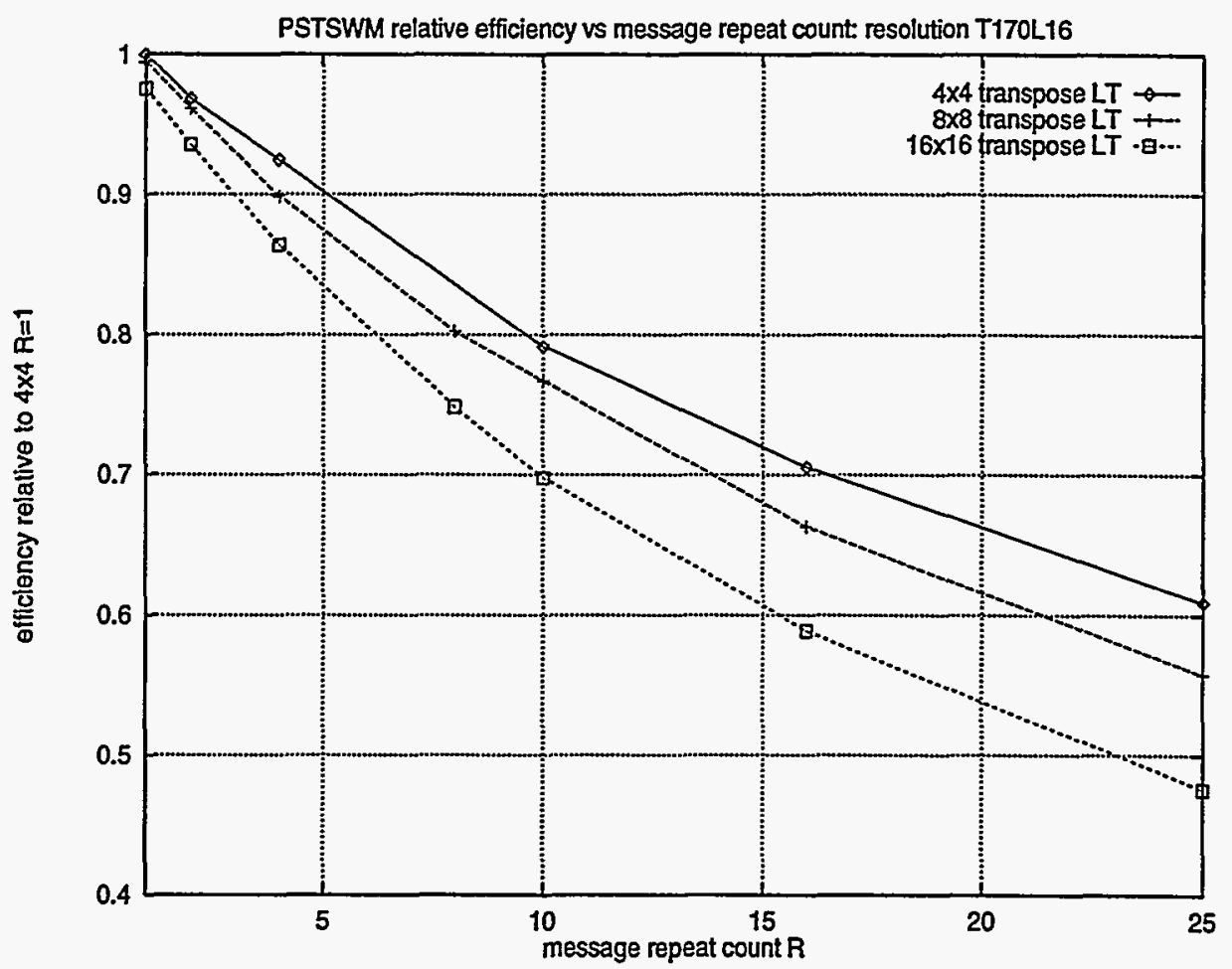

Figure 10: Variation in relative efficiency as message repeat count increases, for PSTSWM at resolution T170L16.

one message repetition. Both the delay test and the message repetition test are plotted on the same graph in Figure 11 for the transpose LT algorithm on a $4 \times 4$ grid. This test demonstrates the need to consider contention when studying the effects of bandwidth. If contention must be considered, then topology is an important issue.

\begin{tabular}{|c|c|c|c|}
\hline$\Delta$ Repeat & $\begin{array}{c}\Delta \text { Time } \\
(\mathrm{sec})\end{array}$ & $\begin{array}{c}\Delta \text { Time } \\
\text { per Message (msec) }\end{array}$ & $\begin{array}{c}\text { Comm. Time } \\
\text { Ratio }\end{array}$ \\
\hline R1-R10 & 113 & 47.56 & 3.73 \\
R10-R32 & 263 & 45.28 & 3.55 \\
R1-R32 & 376 & 45.94 & 3.61 \\
\hline
\end{tabular}

Table 3: Distributed IT: T170L16 $4 \times 4$ grid run times for various $R$ values.

Tables 3 and 4 are attempts to quantify the amount of contention. Unlike PCTH, PSTSWM uses nonblocking communication protocols and contention cannot be measured by timing indiviudal sends. Instead, the ratio of the time to send a message in an echo test versus the time observed in PSTSWM is assumed to represent the average message contention seen by the application The second column of the table is the difference in execution time between different numbers of repeat counts. On the $4 \times 4$ grid 12 messages are sent per time step, and there are 22 time steps. The third column is the difference in the amount of time to send each message. Again, we assume that every message is $2 \mathrm{MB}$ and that it takes 0.012739 seconds to send a message of this length. Column four is formed by dividing column 3 by 12.739 to derive the 


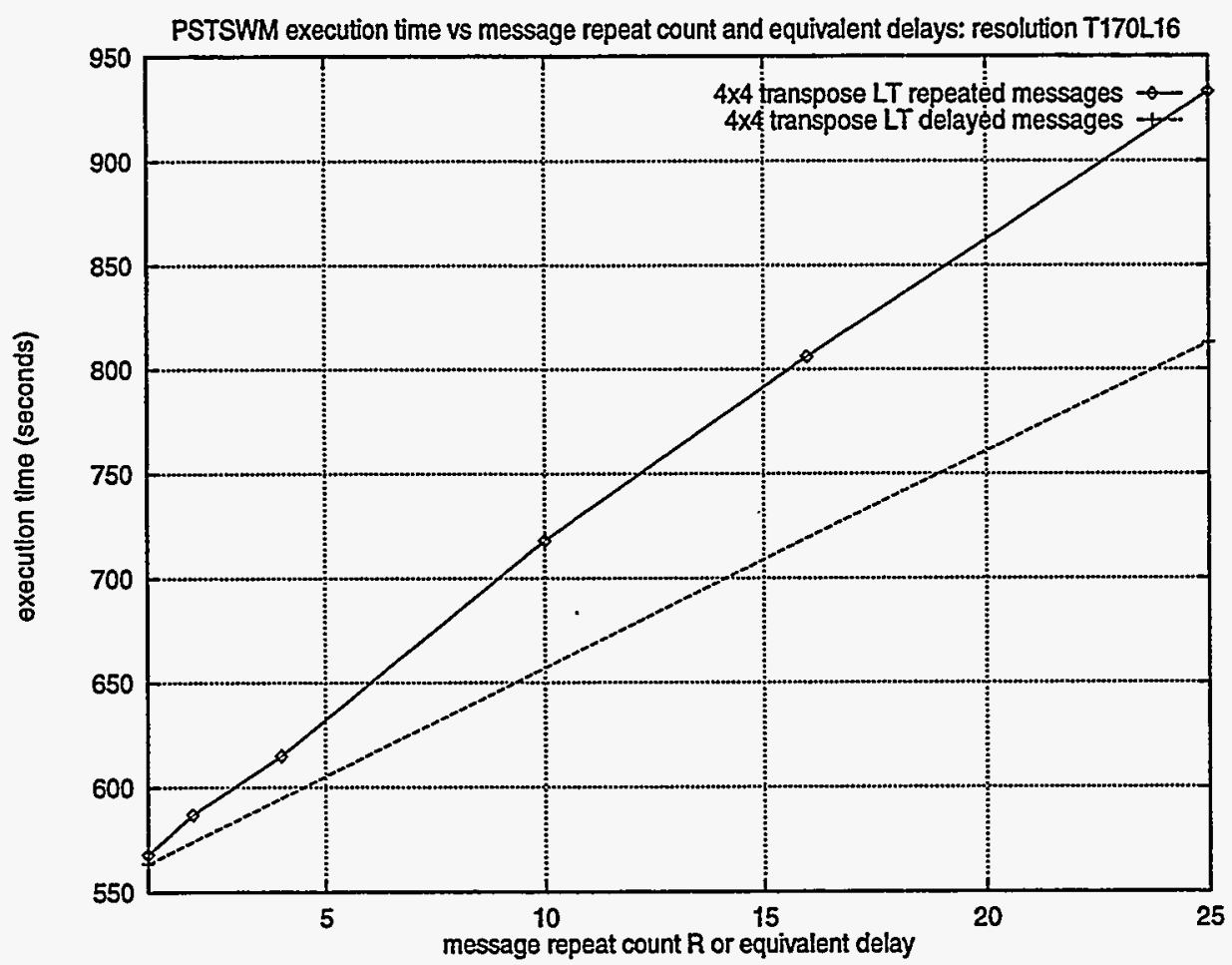

Figure 11: Differences between performance of PSTSWM resolution T170L16 on a $4 \times 4$ grid with repeating messages which preserves mesh contention and equivalent message delays which does not preserve mesh contention.

contention factor for PSTSWM. These results show a contention factor of 4 to 5 , depending on the parallel algorithm used.

\begin{tabular}{|c|c|c|c|}
\hline$\Delta$ Repeat & $\begin{array}{c}\Delta \text { Time } \\
(\mathrm{sec})\end{array}$ & $\begin{array}{c}\Delta \text { Time } \\
\text { per Message (millisec) }\end{array}$ & $\begin{array}{c}\text { Comm. Time } \\
\text { Ratio }\end{array}$ \\
\hline R1-R10 & 150 & 63.1 & 4.96 \\
R10-R25 & 215 & 54.3 & 4.26 \\
R1-R25 & 365 & 57.61 & 4.52 \\
\hline
\end{tabular}

Table 4: Transpose LT: T170L16 4x4 grid run times for various $\mathrm{R}$ values.

Earlier we stated that if contention is important then topology must also be important. In the last experiment we maintained a $8 \times 8$ logical grid, but varied the physical grid from $1 \times 64$ to $64 x 1$. The results are shown in Figure 12 . The optimal physical grid was $16 \times 4$. The $8 \times 8$ grid equalizes the distance messages travel for the FFT and the LT, while the $16 \times 4$ layout decreases the distance messages travels for the FFT at the expense of increasing the message distances in the LT. Since the FFT routines send longer messages than the LT, mapping the $8 \times 8$ logical grid onto a physical $16 \times 4$ grid achieves the optimal performance. Note that it is not the distance per se that affects performance, but rather the number of messages contending for the same physical links, which is increased when the distance increases in these algorithms. This contention is maximized in the $1 \times 64$ and $64 \times 1$ cases, which have the worst performance. 


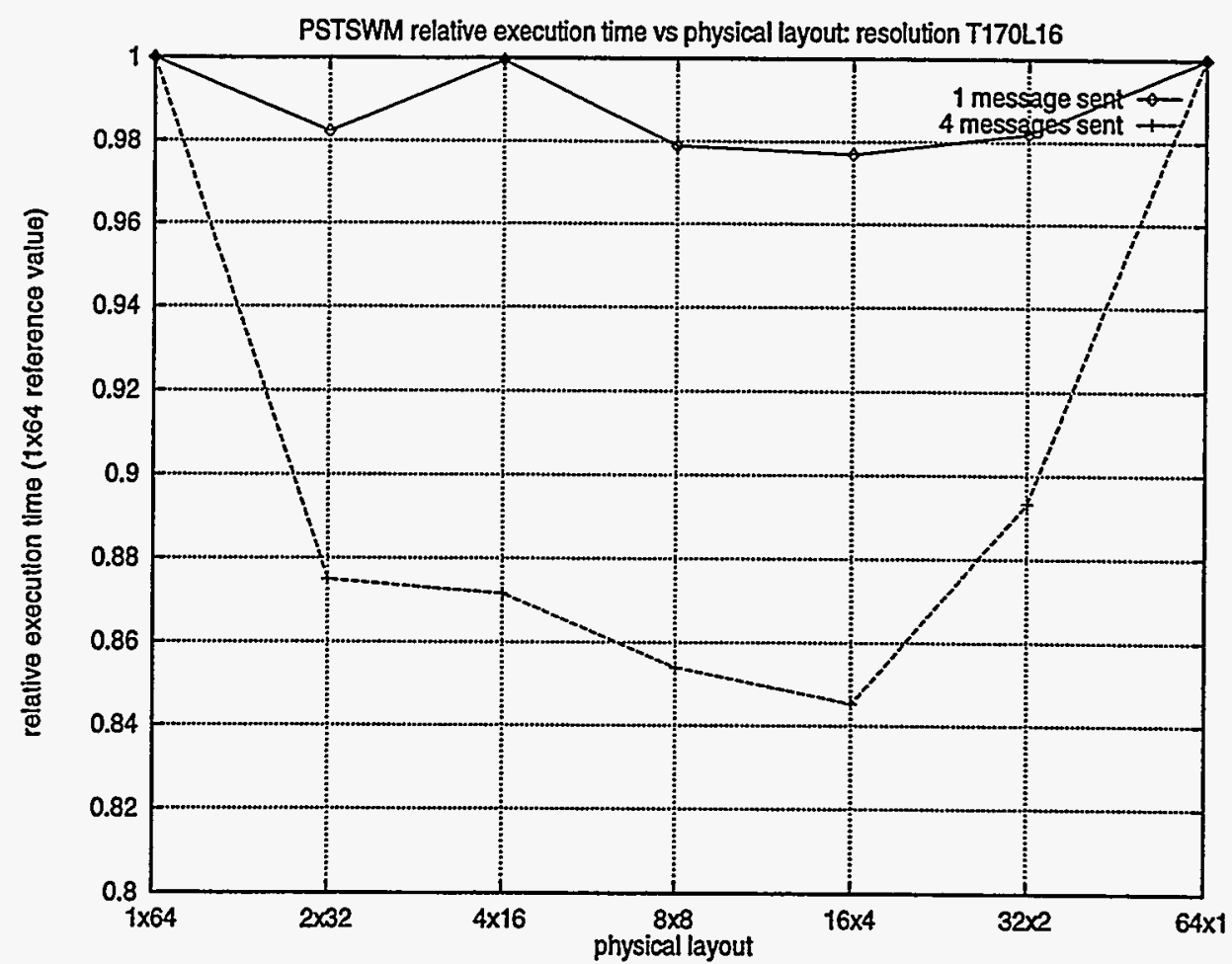

Figure 12: Relative performance of PSTSWM T170L16 logical $8 \times 8$ grid mapped across various 2 dimensional physically layouts.

The example where messages were sent four times demonstrate the importance of the relationship between topology and bandwidth. When each message was sent only once, performance never varied more than $2 \%$. When messages were sent four times, performance varied by over 14\%. The effects of suboptimal topology can be overcome with additional bandwidth, while poor bandwidth emphasizes the problems of topology.

\section{Discussion}

PCTH and PSTSWM differ significantly in their communication requirements, scaling behaviors, and in their modes of operation (fixed problem size vs. scaleup). Consequently, their performance is influenced differently by bandwidth and latency. For example, for PSTSWM on 256 nodes at resolution T170L16, a reduction in effective bandwidth by a factor of 10 resulted in a $50 \%$ increase in execution time. In contrast, for PCTH on 256 nodes at a 1 million cell resolution, a reduction in effective bandwidth by a factor of 8 only resulted in an $11 \%$ increase in execution time. In the scaleup analysis for PCTH, a 16-fold bandwidth reduction on 512 processors only resulted in a $9.5 \%$ increase in execution time.

For codes such as PSTSWM, the requirement for high bandwidth appears to be independent of the number of processors. Figure 10 showed how efficiency decreased as bandwidth decreased for three different numbers of processors. All curves behave similarly, indicating that high 
bandwidth is equally important whether using 16 processors or 256 processors. Thus, for computer architects designing scalable interconnects, bandwidth should be maintained across all supported scales. While some codes are more sensitive to bandwidth than others, we argue that the results of this study indicate that the need for high bandwidth interconnects is still evident.

Design and selection of MPP systems is a balance between processor power, amount of memory, I/O capabilities, and interconnect fabric. It is clear that this balance is quite application dependent. PCTH is tolerant enough that it appears that a decrease in the amount of Paragon memory per node or a decrease in the interconnect bandwidth with an accompanying increase in the number of nodes would improve overall PCTH performance for a given machine cost. On the other hand, PSTSWM execution time increased by $127.8 \%$ on a $16 \times 16$ grid of compute nodes at resolution T170L16 for a message repeat count of 16 so that no decrease in bandwidth can be tolerated. For other parallel machines with slower interconnects even PCTH could be substantially degraded, depending on the available memory of the nodes of the machine. Ethernet runs at a maximum of $1.2 \mathrm{MB} / \mathrm{sec}$, which is 133 times slower than the Paragon interconnect running SUNMOS. Effective Ethernet speeds may be even slower with software overheads. Figure 6 clearly showed a major reduction in execution time for PCTH when effective bandwidth was decreased by this large of a factor. FDDI connections approach $10 \mathrm{MB} / \mathrm{sec}$ which is still a reduction in bandwidth of a factor of 16 with a possible significant reduction in performance. The topological interconnect of these other technologies must also be considered and will clearly have a major impact on performance for large numbers of nodes. The contention evident in the $2 \mathrm{D}$ mesh can only grow for ring or tree type interconnects.

One cannot always control the aspect ratio of the node layout as was done on the Paragon with the PSTSWM tests. "Excess" bandwidth is required to reduce the effect of poor node allocation and inter-job network contention, as may occur when jobs are run via a batch scheduling system such as NQS or if a user must fit his job around jobs already running.

Simulations such as PCTH are set up to run problems which require several hours to several days to complete. Weather forecast codes similar to PSTSWM are intended to run continually and have tight deadlines on the order of a few hours by which each simulation must be complete. For climate models, on the other hand, parameter studies based on 10 or 100 year simulations may require months or years of computing time. Clearly if this much compute time is being consumed on thousands of processors, even a small improvement in runtime is important. Both of the applications studied clearly demonstrate that processor interconnect technology is important when viewed in this light. In the years to come the number of available compute nodes will grow larger and the individual microprocessor performance will improve at the same time as applications are coded to run more efficiently on a single node. All of these effects will 
tend to put additional stress on the ability of the underlying interconnect technology to deliver.

\section{Acknowledgement}

Work on this project at Sandia National Laboratory is funded by the United States Department of Energy (DOE) under Contract DE-AC04-94AL85000. Work on this project at Oak Ridge National Laboratory is funded by DOE under contract DE-AC05-84OR21400. The Intel XP/S 150 MP Paragon operated by the Center for Computational Science at ORNL is funded by the Department of Energy's Mathematical, Information and Computational Sciences Division of the Office of Computational and Technology Research.

\section{References}

[1] J. BRehm, L. Dowdy, M. MadhUKar, AND E. SMIRnI, PerPreT - a performance prediction tool, in Quantitative Evaluation of Computing and Communication Systems, Lecture Notes in Computer Science 977, Springer, Heidelberg, 1995.

[2] G. L. Browning, J. J. HACK, AND P. N. Swarztrauber, A comparison of three numerical methods for solving differential equations on the sphere, Mon. Wea. Rev., 117 (1989), pp. 1058-1075.

[3] J. D. Dongarra and T. H. Dunigan, Message-passing performance of various computers, Tech. Report ORNL/TM-13006, Oak Ridge National Laboratory, Oak Ridge, TN, February 1996.

[4] J. B. Drake, I. T. Foster, J. G. Michalakes, B. Toonen, and P. H. Worley, Design and performance of a scalable parallel community climate model, Parallel Computing, 21 (1995), pp. 1571-1591.

[5] I. T. Foster, B. TOONEn, AND P. H. Worley, Performance of parallel computers for spectral atmospheric models, Tech. Report ORNL/TM-12986, Oak Ridge National Laboratory, Oak Ridge, TN, April 1995. (also, J. Atm. Oceanic Tech accepted).

[6] I. T. FOSTER AND P. H. WORLEY, Parallel algorithms for the spectral transform method, Tech. Report ORNL/TM-12507, Oak Ridge National Laboratory, Oak Ridge, TN, May 1994. (also, SIAM J. Sci. Comput. accepted).

[7] J. J. HaCk, B. A. Boville, B. P. Briegleb, J. T. KieHL, P. J. Rasch, and D. L. Williamson, Description of the NCAR Community Climate Model (CCM2), NCAR Tech. Note NCAR/TN-382+STR, National Center for Atmospheric Research, Boulder, Colo., 1992. 
[8] J. J. HACK AND R. JAKOB, Description of a global shallow water model based on the spectral transform method, NCAR Tech Note NCAR/TN-343+STR, National Center for Atmospheric Research, Boulder, CO, February 1992.

[9] P. HEIDELBERger AND K. S. TRIVEDI, Analytic queuing models for programs with internal concurrency, IEEE Trans. Comput., c-32 (1983), pp. 73-82.

[10] G. LYon, R. KACKer, AND A. LINZ, A scalability test for parallel code, Software: Practise and Experience, 25 (1995), pp. 1299-1314.

[11] G. LYON, R. SNELICK, AND R. KACKER, Synthetic-perturbation tuning of MIMD programs, The Journal of Supercomputing, 8 (1994), pp. 5-28.

[12] A. B. Maccabe, K. S. McCurley, R. Riesen, and S. R. Wheat, SUNMOS for the Intel Paragon: A brief users guide, in Proceedings of the Intel Supercomputer Users' Group 1994, Intel Supercomputer Users' Group, 1994, pp. 245-251.

[13] B. MachenhaUer, The spectral method, in Numerical Methods Used in Atmospheric Models, vol. II of GARP Pub. Ser. No. 17. JOC, World Meteorological Organization, Geneva, Switzerland, 1979, ch. 3, pp. 121-275.

[14] J. M. McGlaun, S. L. Thompson, and M. G. ElRICK, CTH: A three dimensional shock wave physics code, Int. J. of Impact Engineering, 10 (1990), pp. 351-360.

[15] J. Painter, P. McCormick, M. Krogh, C. Hansen, and G. C. DE Verdíne, The ACL message passing library, EPFL Supercomputing Review, 7 (1995).

[16] A. C. Robinson and C. T. Vaughan, Block grid application, in High Performance Computing, G. Sabot, ed., Addison-Wesley Publishing Company, Reading, Massachusetts, 1995, ch. 2.

[17] E. RotHBERG, Characterization of paragon performance benchmarks. Intel Internal Correspondence Memo, February 1994.

[18] — Communication characteristics of parallel scientific applications. Intel Internal Correspondence Memo, August 1994.

[19] A. ThọmASIAN AND P. F. BAY, Analytic queuing network models for parallel processing of task systems, IEEE Trans. Comput., c-35 (1986), pp. 1045-1054.

[20] H. WABNIG AND G. HARING, PAPS - the parallel program performance prediction toolset, in 7th International Conference on Modeling Techniques and Tools for Computer Performance Evaluation, 1994, pp. 284-304. 
[21] S. R. Wheat, A. B. Maccabe, R. Riesen, D. W. van Dresser, and T. M. StallCuP, An operating system for massively parallel systems, in Proceedings of the Twenty Seventh Annual Hawaii International Conference on System Sciences, IEEE Computer Society Press, Los Alamitos, CA, 1994, pp. 56-65.

[22] D. L. Williamson, J. B. Drake, J. J. Hack, R. Jakob, and P. N. Swarztrauber, A standard test set for numerical approximations to the shallow water equations on the sphere, J. Computational Physics, 102 (1992), pp. 211-224.

[23] P. H. Worley and B. Toonen, A users' guide to PSTSWM, Tech. Report ORNL/TM12779, Oak Ridge National Laboratory, Oak Ridge, TN, July 1995. 


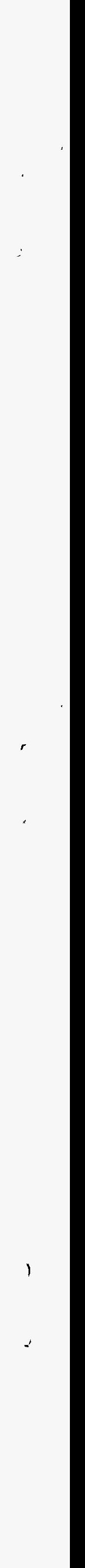


ORNL/TM-13250

\section{INTERNAL DISTRIBUTION}

1. E. F. D'Azevedo

2. T. S. Darland

3. J. J. Dongarra

4. J. B. Drake

5. T. H. Dunigan

6. G. A. Geist

7. K. L. Kliewer

8-12. M. R. Leuze

13. D. R. Mackay

14. C. E. Oliver

15. P. M. Papadopoulos
16-20. S. A. Raby

21-25. R. F. Sincovec

26. P. H. Worley

27. Central Research Library

28. ORNL Patent Office

29. K-25 Applied Technology Library

30. Y-12 Technical Library

31. Laboratory Records - RC

32-33. Laboratory Records Department

\section{EXTERNAL DISTRIBUTION}

34. David H. Bailey, NASA Ames, Mail Stop 258-5, NASA Ames Research Center, Moffet Field, CA 94035

35. Ted Barragy, Intel Corp, CO1-02, 15201 NW Greenbrier Pkwy, Beaverton, OR 97006

36. Edward H. Barsis, Computer Science and Mathematics, P. O. Box 5800, Sandia National Laboratory, Albuquerque, NM 87185

37. Donna Bergmark, 745 E \& TC Building, Hoy Road, Cornell University, Ithaca, NY 14853

38. Roger W. Brockett, Wang Professor of EE and CS, Division of Applied Sciences, 29 Oxford Street, Harvard University, Cambridge, MA 02138

39. Bill L. Buzbee, Scientific Computing Division, National Center for Atmospheric Research, P. O. Box 3000, Boulder, CO 80307

40. Jagdish Chandra, Army Research Office, P. O. Box 12211, Research Triangle Park, NC 27709-2211

41. Melvyn Ciment, Deputy Assistant Director Computer and Information Science and Engineering (CISE) National Science Foundation 4201 Wilson Blvd. Arlington, VA 22230

42. Larry Dowdy, Computer Science Department, Vanderbilt University, Nashville, TN 37235

43. Geoffrey C. Fox, NPAC, 111 College Place, Syracuse University, Syracuse, NY $13244-4100$

44. Dennis B. Gannon, Computer Science Department, Indiana University, Bloomington, IN 47401 
45. Alan George, Department of Computer Science, University of Waterloo, Waterloo, Ontario, Canada N2L 3G1

46. Gene Golub, Computer Science Department, Stanford University, Stanford, CA 94305

47. Eric Grosse, Bell Laboratories 2T-504, Murray Hill, NJ 07974

48. John L. Gustafson, Ames Laboratory, 236 Wilhelm Hall, Iowa State University, Ames, IA 50011-3020

49. Michael T. Heath, Department of Computer Science, 4157 Beckman Institute 405 North Mathews, Urbana, IL 61801

50. John L. Hennessy, CIS 208, Stanford University, Stanford, CA 94305

51. Anthony J. G. Hey, Dept. of Electronics and Computer Science, University of Southampton, Highfield, Southampton SO9 5NH, United Kingdom.

52. Dan Hitchcock, ER-31, Mathematical, Information, and Computational Sciences Division, Office of Computational and Technology Research, Office of Energy Research, U.S. Department of Energy, Washington, DC 20585

53. Charles J. Holland, Air Force Office of Scientific Research, 110 Duncan Avenue, Suite B115, Bolling Air Force Base, Washington, DC 20332-0001

54. Kenneth Kennedy, Department of Computer Science, Rice University, P.O. Box 1892, Houston, TX 77001

55. Tom Kitchens, ER-31, Mathematical, Information, and Computational Sciences Division, Office of Computational and Technology Research, Office of Energy Research, Washington, DC 20585

56. Richard Lau, Office of Naval Research, Code 111MA 800 Quincy Street, Boston Tower 1, Arlington, VA 22217-5000

57. Peter D. Lax, Courant Institute of Mathematical Sciences, New York University, 251 Mercer Street, New York, NY 10012

58. E. D. Lazowska, Department of Computer Science and Engineering, Sieg Hall, FR-35, University of Washington, Seattle, WA 98195

59. Ted Lewis, Computer Science Department, Naval Postgraduate School, code 013, 1 University Circle, Monterey, CA 93943-5001

60. Rik Littlefield, Pacific Northwest Laboratory, MS K1-87, P.O.Box 999, Richland, WA 99352

61. James McGraw, Lawrence Livermore National Laboratory, L-306, P. O. Box 808, Livermore, CA 94550

62. Barton P. Miller, Computer Sciences Department, University of Wisconsin - Madison, 1210 West Dayton Street, Madison, WI 53706

63. David B. Nelson, Associate, Director, Office of Computational and Technology Research, ER-30, Office of Energy Research, U.S. Department of Energy, Washington, DC 20585 
64. Joseph Oliger, Computer Science Department, Stanford University, Stanford, CA 94305

65. James M. Ortega, Department of Applied Mathematics, Thornton Hall, University of Virginia, Charlottesville, VA 22901

66. Linda R. Petzold, Computer Science Department, University of Minnesota, 200 Union Street, S.E., Room 4-192, Minneapolis, MN 55455

67. James C. T. Pool, Deputy Director, Caltech Concurrent Supercomputing Facility, California Institute of Technology, MS 158-79, Pasadena, CA 91125

68. Daniel A. Reed, Computer Science Department, University of Illinois, Urbana, IL 61801

69. Allen C. Robinson, Sandia National Laboratories, Computational Physics Research \& Development, 1431, P.O. Box 5800, Albuquerque, NM 87185-0819

70. Diane T. Rover, 155 Engineering Building, Department of Electrical Engineering, Michigan State University, East Lansing MI 48824

71. Ahmed H. Sameh, Department of Computer Science, University of Minnesota, 200 Union Street S.E., Minneapolis, MN 55455

72. Charles L. Seitz, Department of Computer Science, California Institute of Technology, Pasadena, CA 91125

73. Giuseppe Serazzi, Politecnico di Milano, Dipartimento di Elettronica e Informazione, P.zza L. da Vinci 32, I 20133 Milano, Italy

74. Kenneth C. Sevcik, Computer Systems Research Institute, 10 King's College Road, University of Toronto, Toronto, Ontario M5S 1A1, Canada

75. Margaret L. Simmons, Computing and Communications Division, Los Alamos National Laboratory, Los Alamos, NM 87545

76. Horst D. Simon, Director, NERSC Division, Lawrence Berkeley National Laboratory, Mail Stop 50A/5104, University of California, Berkeley, CA 94720

77. Burton Smith, Tera Computer Company, 2815 Eastlake Ave East, Seattle, WA 98102

78. Marc Snir, IBM T.J. Watson Research Center, Department 420/36-241, P. O. Box 218, Yorktown Heights, NY 10598

79. Rick Stevens, Mathematics and Computer Science Division, Argonne National Laboratory, 9700 South Cass Avenue, Argonne, IL 60439

80. Paul N. Swarztrauber, National Center for Atmospheric Research, P. O. Box 3000, Boulder, CO 80307

81. Andrew B. White, Computing Division, Los Alamos National Laboratory, Los Alamos, NM 87545

82. Office of Assistant Manager for Energy Research and Development, U.S. Department of Energy, Oak Ridge Operations Office, P. O. Box 2001, Oak Ridge, TN $37831-8600$

83-84. Office of Scientific \& Technical Information, P. O. Box 62, Oak Ridge, TN 37831 